\title{
GIẢI PHÁP VÀ KỸ THUẬT TRIỄN KHAI ỨNG DỤNG MÔ HÌNH KẾ TOÁN QUẢN TR! ABC (ACTIVITY BASED COSTING) CHO NHÓM CÁC DOANH NGHIÊP KHAI THÁC CẢNG CỦA VIỆT NAM
}

\author{
TRẦ THÚ' BA, LÊ THI THU TRANG \\ Khoa Kế toán Kiểm toán, Trương Đại học Công nghiệp thành phố Hồ Chi Minh; \\ tranthuba@iuh.edu.vn
}

Tóm tắt. Bài viết trình bày kết quả nghiên cứu các nhân tố ảnh hưởng đến việc triển khai \& ứng dụng mô hình kế toán quản trị theo phương pháp $\mathrm{ABC}$ (Activity Based Costing) làm cơ sở để tính giá thành hợp lý của dịch vụ cảng biển đối với nhóm doanh nghiệp khai thác Cảng tại Việt Nam. Căn cứ kết quả nghiên cứu Tác giả đề xuất một số giải pháp cũng như kỹ thuật triển khai và vận dụng mô hình kế toán quản trị $\mathrm{ABC} / \mathrm{TD} . \mathrm{ABC}$ (Time - Driven Activity Based Costing) giúp cho nhóm các doanh nghiệp khai thác Cảng của Việt Nam có thể triển khai và vận dụng thành công mô hình kế toán quản trị $\mathrm{ABC} / \mathrm{TD} . \mathrm{ABC}$. Kết quả nghiên cứu thực nghiệm với dữ liệu khảo sát của 211 mẫu hợp lý của các doanh nghiệp khai thác Cảng cho thấy việc ứng dụng mô hình kế toán quản trị $\mathrm{ABC}$ bị tác động bởi các yếu tố như: Cơ cấu tổ chức, Sự ủng hộ của lãnh đạo, Hạn chế kỹ thuật, Nhận thức, Nguồn lực, Tâm lý nhân viên, Xây dựng mục tiêu. Trong đó, đặc biệt hai nhân tố có trọng số ảnh hưởng rất cao là sụ ủng hộ của lãnh đạo (0.630) và hạn chế kỹ thuật (0.578).

Từ khóa. Phương pháp $\mathrm{ABC}$, kế toán quản trị, nhân tố ảnh hưởng.

\section{SOLUTIONS AND TECHNIQUES PERFORM TO APPLY THE ABC MANAGEMENT ACCOUNTING MODEL INTO PORT OPERATING COMPANIES IN VIETNAM}

\begin{abstract}
The paper presents the result of research on the factors affecting the implementation and application of the activity based costing (ABC) accounting model as the basis for calculating the reasonable cost of seaport service at enterprises exploiting ports of vietnam. Based on the research results, the authors propose some solutions as well as techniques for implementing and applying the $\mathrm{ABC} / \mathrm{TD} . \mathrm{ABC}$ (time-driven activity based costing) management accounting model to help the port exploited enterprises can deploy and successfully apply management accounting ABC/TD.ABC. The results of empirical research with survey data of 211 observes from port exploited enterprises show that the application of the $\mathrm{ABC}$ management accounting model is influenced by factors such as organizational structure, leadership support, technical constraints, awareness, resources, staff psychology, goal setting. In particular, two factors with very high weight are the support of leaders (0.630) and technical limitations (0.578).
\end{abstract}

Key words. $\mathrm{ABC}$ method, management accounting, impcat factor.

\section{GIỚI THIỆ}

Công tác kế toán đa phần tại các doanh nghiệp Việt Nam hiện nay chủ yếu phục vụ công tác báo cáo tài chính và báo báo thuế cho các đối tượng bên ngoài. Trong khi ấy với xu thế hội nhập, cạnh tranh công bằng thì không còn khái niệm ô che của chính phủ, vì vậy đòi hỏi các doanh nghiệp trong nước phải tự vận động. Để làm được điều đó, các doanh nghiệp Việt Nam phải thay đổi tư duy về kế toán. Sử dụng dữ liệu của kế toán để quản trị, đó mới là vai trò thật sự của kế toán hiện đại. Một phương pháp mới của kế toán quản trị ở Việt Nam đó là phương pháp ABC (Activity Based Costing) hoặc TD.ABC (Time Driven Activity Based Costing). Nguyên tắc của phương pháp này dựa trên nguyên nhân hình thành chi phí làm cơ sở để lựa chọn tiêu thức phân bổ hợp lý với dòng chi phí chung phát sinh để tính vào giá thành. Chính vì thế, phương pháp $\mathrm{ABC}$ khắc phục những nhược điểm còn tồn tại trong phương pháp truyền thống. 
Mặc khác, trong thời đại hội nhập bên cạnh những lợi thế thì thách thức đặt ra với các doanh nghiệp trong nước nói chung và nhóm doanh nghiệp khai thác Cảng nói riêng là làm thế nào để nâng cao khả năng cạnh tranh với các nhóm đối thủ mạnh trên thế giời. Đặc biệt hơn, nhóm ngành khai thác Cảng Nhóm ngành đóng góp khá lớn tới nền kinh tế của nước ta hiện nay vẫn còn những bất cập trong cách quản lý cũng như tư duy về kế toán vẫn còn mang tính truyền thống đơn giản. Trong khi ây, môi trường và tính chất công việc ngày càng mang tính vĩ mô, phức tạp hơn.

Vì vậy, thực tế đặt ra là cần có giải pháp thay đổi trong cách quản lý của các nhóm doanh nghiệp này. Đó cũng là lý do tác giả chọn đề tài nghiên cứu phương pháp ứng dụng mô hình kế toán quản trị $\mathrm{ABC}$ nhằm tìm ra những nhân tố ảnh hưởng tới công tác triển khai đối với nhóm doanh nghiệp khai thác Cảng. Từ đó, đề xuất các giải pháp cũng như kỹ thuật triển khai ứng dụng mô hình kế toán quản trị $\mathrm{ABC} / \mathrm{TD} . \mathrm{ABC}$ thành công nhằm nâng cao năng lực quản lý tài chính và tính cạnh tranh của các doanh nghiệp khai thác cảng của Việt nam.

\section{CƠ SỞ LÝ THUYÊTT \& MÔ HÌNH NGHIÊN CỨU}

\subsection{Cơ sở lý thuyết về phương pháp $\mathrm{ABC}$}

Quá trình phát triển hệ thống kế toán chi phí trải qua bốn giai đoạn [1]. Trong đó, giai đoạn hai hệ thống kế toán chi phí đã giải quyết được vấn đề định giá hàng tồn kho để phục vụ lập báo cáo tài chính định kỳ. Với mục đích định giá hàng tồn kho, các nguyên tắc kế toán tài chính không yêu cầu ấn định chi phí sản xuất chung theo mối quan hệ nhân quả dựa trên nhu cầu của từng sản phẩm với mỗi nguồn lực sử dụng. Rất nhiều hệ thống kế toán chi phí trong giai đoạn này phân bổ chi phí gián tiếp theo cách đơn giản như dựa trên tiêu thức lao động trực tiếp, sản lượng, giá trị nguyên vật liệu, giờ máy chạy. Các tiêu thức này phù hợp khi tỷ trọng giá trị của lao động, nguyên vật liệu chiếm giá trị lớn trong giá thành sản phẩm. Phương pháp này được gọi là phương pháp đánh giá chi phí truyền thống (Traditional cost system TCS). Tuy nhiên, các chi phí gián tiếp dần có tỷ trọng lớn trong giá trị sản phẩm và quá trình sản xuất. Hơn nữa, theo phân loại chi phí trong giai đoạn này có một số chi phí được ghi nhận là chi phí bán hàng, chi phí cho kênh phân phối, chi phí quản lý doanh nghiệp, chi phí nghiên cứu phát triển được ghi nhận là chi phí thời kỳ, thực chất các chi phí này liên quan đến sản phẩm và khách hàng, nhưng các chi phí này không được phân bổ vào các đối tượng này. Phương pháp chi phí truyền thống bóp méo chi phí sản phẩm, chi phí dịch vụ, đưa ra thông tin về chi phí thấp hơn thực tế đối với các quy trình sản xuất phức tạp hay cao hơn thực tế đối với quy trình sản xuất đơn giản [4\&5]. Mô hình phân bổ chi phí dựa trên hoạt động (Activity - Based Costing, $\mathrm{ABC}$ ) được xây dựng để giải quyết nhược điểm của TCS về phân bồ chi phí chính xác đến sản phẩm và khách hàng. Đồng thời $\mathrm{ABC}$ giúp cung cấp thông tin cho hệ thống kiểm soát điều hành và học hỏi của công ty [1]

Một số quan điểm và khái niệm về phuơng pháp $A B C$

"Phương pháp phân bổ chi phí dựa trên hoạt động $(\mathrm{ABC})$ là một khái niệm về kế toán chi phí, khái niệm dựa trên tiền đề sản phẩm sử dụng các hoạt động để hoàn thành, và các hoạt động đòi hỏi các chi phí để thực hiện. Như vậy, hệ thống này được thiết kế các chi phí không được tập hợp trực tiếp đến các sản phẩm, mà các chi phí được tập hợp đến các hoạt động, tiếp theo các hoạt động được tập hợp đến các sản phẩm tiêu dùng trực tiếp hoạt động đó" [7]

Theo Turney (1996) mô hình $\mathrm{ABC}$ là một quá trình của kế toán quản trị nhằm phân bổ các chi phí nguồn lực đến sản phẩm, khách hàng dựa trên các hoạt động. Theo Kaplan (1998) mô hình $\mathrm{ABC}$ là một bản đồ kinh tế mô tả các chi phí và lợi nhuận trong công ty dựa trên khái niệm về các hoạt động của công ty. Mô hình $\mathrm{ABC}$ dùng để trả lời cho các câu hỏi sau:

+ Những hoạt động gì đang được tiến hành và sử dụng nguồn lực của công ty.

+ Chi phí thực hiện các hoạt động là bao nhiêu và cho toàn quá trình kinh doanh là bao nhiêu.

+ Tại sao công ty lại cần các hoạt động hay quá trình kinh doanh này.

+ Mỗi nguồn lực tham vào vào sản phẩm và dịch vụ của công ty là bao nhiêu.

Theo Robin (2009) ABC được định nghĩa là một hệ thống kể toán ghi nhận chi phí theo biến đổi của hoạt động thực hiện trong đơn vị và sử dụng nhiều tiêu thức để phân bổ chi phí sản xuất chung đến các sản phẩm. Nguyên tắc chính của phương pháp này là dựa vào các hoạt động chuyển hoá các nguồn lực thành các đầu ra của doanh nghiệp. 


\section{GIẢI PHÁP VÀ KỸ THUẬT TRIỂN KHAI ÚNG DỤNG MÔ HİNH KẾ TOÁN QUẢN TRI 21 ABC (ACTIVITY BASED COSTING) CHO NHÓM CÁC DOANH NGHIÊP KHAI THÁC CẢNG CỦA VIỆT NAM}

Theo Brent (2012) ABC là phương pháp phân bổ chi phí chính xác đến hoạt động, quá trình sản xuất, sản phẩm, dịch vụ, khách hàng, lĩnh vực kinh doanh của công ty. Phương pháp này dựa trên khái niệm là các nguồn lực dùng để sản xuất sản phẩm và dịch vụ đều có thể lượng hoá và phân bổ đến các sản phẩm và dịch vụ [10]. Do đó, Johnson (1990) nhận định rằng $\mathrm{ABC}$ là một trong hai hay ba phát minh quan trọng nhất của kế toán quản trị của thế kỷ 20 .

Nhu vậy, phuoong pháp ABC là phương pháp phân bổ chi phí sủ dụng đa tiêu thức để phân bổ chi phí đến đối tương cuối cùng là sản phâm, khách hàng, thị truờng, kênh phân phối... Nguyên tắc chính của phuơng pháp là tính chi phi cho tìng hoạt động, sau đó tổng hợp các chi phí đến đối tượng cuối cùng bằng cách đo luờng bao nhiêu hoạt động phục vu cho đối tượng này.

Kaplan \& Roobin (1998) cho rằng: Phương pháp $\mathrm{ABC}$ được thiết kế nhằm cung cấp một cách thức phân bổ chính xác chi phí gián tiếp và nguồn lực cho các hoạt động, quá trình kinh doanh, các sản phẩm và dịch vụ. Mục tiêu của phương pháp $\mathrm{ABC}$ không phải là phân bổ chi phí chung cho các sản phẩm mà mục tiêu của phương pháp $\mathrm{ABC}$ là đo lường và tính giá tất cả các nguồn lực sử dụng cho các hoạt động mà có thể hỗ trợ cho việc sản xuất và phân phối sản phẩm, dịch vụ đến với khách hàng [1]. Theo Sirirat (2010), các nghiên cứu về $\mathrm{ABC}$ liên quan đến các công ty có quy mô lớn hơn là các công ty vừa và nhỏ. Rất ít kinh nghiệm triển khai hay có nghiên cứu về các công cụ thích hợp ứng dụng cho các công ty thuộc quy mô vừa và nhỏ này. Owen (2011) tiến hành nghiên cứu mô hình $\mathrm{ABC}$ áp dụng vào doanh nghiệp vừa và nhỏ còn rất thấp. Nghiên cứu cho rằng các doanh nghiệp này bị giới hạn vốn, nhân sự, tài chính, công nghệ... Tuy $\mathrm{ABC}$ có thể giúp giảm chi phí, tăng năng suất, hay định giá tốt hơn [3].

Những nghiên cứu trong nước như: Bùi Minh Nguyệt (2013) đã vận dụng phương pháp tính giá trên cơ sở hoạt động $(\mathrm{ABC})$ để phục vụ cho việc ra quyết định chiến lược tại công ty TNHH Ý Chí Việt [11]. Nguyễn Việt Hưng \& Trần Thứ Ba (2016) nghiên cứu khám phá các nhân tố ảnh hưởng đến sự hạn chế vận dụng mô hình $\mathrm{ABC}$ trong kế toán quản trị tại các doanh nghiêp Việt Nam. Bài viết đã đưa ra những cụ thể vể các yếu tố ảnh hưởng tới việc hạn chế vận dụng mô hình kế toán quản trị $\mathrm{ABC}$ tại Việt Nam, đồng thời trình bày cụ thể về cơ sở lý luận liên quan tới nội dung nghiên cứu. Một số nghiên cứu về phương pháp ABC của các tác giả khác như Nguyễn Thị Ngọc Lan (2012), Phạm Hồng Hải (2013), Nguyễn Huy Tâm(2013), các nghiên cứu so sánh phương pháp $\mathrm{ABC}$ và phương pháp tính giá truyền thống - Sự cần thiết áp dụng phương pháp $\mathrm{ABC}$ cho các doanh nghiệp Việt $\mathrm{Nam}$, đặc biệt là bài viết của các tác giả Huỳnh Tấn Dũng (2013).

\subsection{Mô hình nghiên cứu}

Các nhân tố ảnh hưởng đến việc vận dụng mô hình kế toán quản trị theo phương pháp $\mathrm{ABC}$ tại các doanh nghiệp được Tác giả tổng hợp dựa trên các nghiên cứu trước có liên quan $\mathrm{ABC}$ (Bảng 1). 
22 GIẢI PHÁP VÀ KỸ THUẬT TRIỂN KHAI ỨNG DỤNG MÔ HİNH KẾ TOÁN QUẢN TRI ABC (ACTIVITY BASED COSTING) CHO NHÓM CÁC DOANH NGHIÊP KHAI THÁC CẢNG CỦA VIỆT NAM

Bảng 1. Tổng hợp các nhân tố ảnh hưởng tới việc vận dụng mô hình ABC tại các doanh nghiệp

\begin{tabular}{|c|c|c|}
\hline Biến & Nhân tố ảnh hưởng & Nghiên cứu của \\
\hline ССТC & $\begin{array}{l}\text { Cơ cấu tổ chức chưa thay đồi phù hợp để } \\
\text { triển khai mô hình } \mathrm{ABC}\end{array}$ & $\begin{array}{l}\text { Wiriya (2002) Manivannan (2010), Suzanne (2011), } \\
\text { Sorinel (2012). }\end{array}$ \\
\hline UHCC & Thiếu sự ủng hộ của lãnh đạo & $\begin{array}{l}\text { Robin (2009), Rosebud (2014). Nguyễn Việt Hưng\&Trần } \\
\text { Thứ Ba (2016) }\end{array}$ \\
\hline HCKT & Hạn chế về kỹ thuật vận dụng & $\begin{array}{l}\text { Kaplan (2007), Richard (2007), Devinaga (2011), } \\
\text { Rosebud (2013). Nguyễn Thị Ngọc Lan (2013). Nguyễn } \\
\text { Việt Hưng\&Trần Thứ Ba(2016) }\end{array}$ \\
\hline CLKD & $\begin{array}{l}\text { Chưa xây dựng chiến lược kinh doanh đòi } \\
\text { hỏi sử dụng mô hình } \mathrm{ABC}\end{array}$ & $\begin{array}{l}\text { Kaplan (1998), Owen (2011), Devinaga (2011), Djordje } \\
\text { (2013), Phillip (2013), Dũng (2014). }\end{array}$ \\
\hline NL & Thiếu nguồn lực & $\begin{array}{l}\text { Amrik (1998), (Nguyễn Thị Ngọc Lan (2013) Nguyễn } \\
\text { Việt Hưng\&Trần Thứ Ba (2016) }\end{array}$ \\
\hline TLNV & Tâm lý hạn chế thay đổi & $\begin{array}{l}\text { Brent (2012), Phillip (2013) Rosebud (2013), Henrik } \\
\text { (2011), Hà (2011). Nguyễn Thị Ngọc Lan (2013) Nguyễn } \\
\text { Việt Hưng\&Trần Thứ Ba (2016) }\end{array}$ \\
\hline XDMT & $\begin{array}{l}\text { Không xây dựng mục tiêu cho từng giai } \\
\text { đoạn triển khai gắn với khen thưởng hợp } \\
\text { lý }\end{array}$ & $\begin{array}{l}\text { Teemu (1997), Douglas (2013), Faudziah (2012) Nguyễn } \\
\text { Việt Hưng\&Trần Thứ Ba (2016) }\end{array}$ \\
\hline NT & Hạn chế về nhận thức & $\begin{array}{l}\text { Teemu (1997), Jamaliah (2008); Suzanne (2011), } \\
\text { Faudziah (2012), Phillip (2013) }\end{array}$ \\
\hline $\mathrm{Y}$ & $\begin{array}{l}\text { Hạn chế vận dụng mô hình } \mathrm{ABC} \text { vào các } \\
\text { doanh nghiệp }\end{array}$ & $\begin{array}{l}\text { Kaplan (1998), Linh (2010), Gary (2012), Sorinal (2012), } \\
\text { Nguyệt (2013), Douglas (2013), Nhi (2014). Nguyễn Thị } \\
\text { Ngọc Lan (2013). Nguyễn Việt Hưng\&Trần Thứ Ba } \\
\text { (2016) }\end{array}$ \\
\hline
\end{tabular}

Nguồn: Tác giả tổng hợp

\section{Mô hình nghiên cứu}

Căn cứ theo các kết quả nghiên cứu của các tác giả trong và ngoài nước (bảng 1). Tác giả đề xuất mô hình nghiên cứu các nhân tố ảnh hưởng đến việc vận dụng mô hình kế toán quản trị theo phương pháp $\mathrm{ABC}$ trong các doanh nghiệp khai thác Cảng của Việt Nam như sau:

$$
\mathrm{Y}=\mathrm{a}_{0}+\mathrm{a}_{1} \mathrm{CCTC}+\mathrm{a}_{2} \mathrm{UHCC}+\mathrm{a}_{3} \mathrm{HCKT}+\mathrm{a}_{4} \mathrm{CLKD}+\mathrm{a}_{5} \mathrm{NL}+\mathrm{a}_{6} \mathrm{TLNV}+\mathrm{a}_{7} \mathrm{XDMT}+\mathrm{a}_{8} \mathrm{NT}+\mathrm{e}
$$

Trong đó, các biến độc lập (CCTC, UHCC, HCKT, CLKD, NL, TLNV, XDMT, NT) và biến phụ thuộc $\mathrm{Y}$ được đại diện cho các thành phần (hay nhân tố) như bảng trên, e là sai số (phần dư) của mô hình. Các thang đo (biến quan sát) được thiết kế theo thang đo Likert 5 mức độ: 1-Rất không đồng ý (hoặc rất không hài lòng) đến 5-Rất đồng ý (hoặc rất hài lòng).

\section{PHƯƠNG PHÁP NGHIÊN CÚ̉U}

\subsection{Phương pháp định tính}

Đề tài nghiên cứu sử dụng phương pháp định tính để xác định lại các yếu tố ảnh hưởng đến hạn chế vận dụng phương pháp $\mathrm{ABC}$ trong nhóm doanh nghiệp khai thác Cảng tại Việt Nam, dựa trên sự tham khảo ý kiến của các chuyên gia là các trưởng phòng tài chính kế toán, kể toán trưởng tại các doanh nghiệp trực thuộc tổng Cty Tân Cảng Sài Gòn và giảng viên khoa Kế Toán-Kiểm Toán trường Đại Học Công Nghiệp để nhận diện những nhân tố ảnh hưởng tới việc vận dụng mô hình kế toán quản trị $\mathrm{ABC}$ vào nhóm doanh nghiệp khai thác Cảng tại Việt Nam. Đồng thời thực hiện thảo luận nhóm để xây dựng các khái niệm và đề xuất mô hình nghiên cứu. 


\subsection{Phương pháp định lượng}

Phương pháp định lượng được sử dụng trong nghiên cứu thực hiện các tác vụ như: tổng hợp xử lý thông tin khảo sát trên phần mềm Excel, sau đó nhập vào phần mềm SPSS để thực hiện các phân tích như: Cronbach's alpha, phân tích nhân tố khám phá EFA, phân tích hồi quy đa biến và cuối cùng là phân tích để xem xét hiện tượng tự tương quan nhiễu, phương sai thay đổi.

Phương pháp khảo sát định lượng chính thức được tiến hành từ đầu tháng $5 / 2017$ đến cuối tháng 6/2017, đối tượng chọn mẫu là giám đốc, kế toán trưởng hoặc trưởng/phó phòng tài chính kế toán, nhân viên kế toán quản trị, nhân viên kế toán tài chính (tổng hợp) của các đơn vị trực thuộc Tổng Cty Tân Cảng Sài Gòn. Nghiên cứu định lượng này được thực hiện thông qua phương pháp trực tiếp phát phiếu khảo sát (bảng câu hỏi) kết hợp với gửi qua email (bảng hỏi thiết kê trên google.docs). Mô hình đo lường gồm 42 biến quan sát (nghiên cứu sơ bộ), theo Hair \& Ctg (1998), kích thước mẫu cần thiết là $\mathrm{n}=210$ (42 $\mathrm{x} 5$ ). Để đạt được kích thước mẫu đề ra, 300 phiếu khảo sát được gửi phỏng vấn, kết quả thu về được 235 phiếu, trong đó có 211 phiếu hợp lệ. Dữ liệu được tổng hợp và phân tích trên 2 phần mềm Excel 2013, SPSS 22.

\section{KẾT QUẢ NGHIÊN CÚ̉U}

\subsection{Nghiên cứu thực trạng doanh nghiệp khai thác cảng tại Việt Nam}

\section{Đặc điểm của doanh nghiệp khai thác cảng}

Khai thác cảng là quá trình tiến hành hoạt động sản xuất kinh doanh, đảm bảo hàng hóa thông qua cảng trong điều kiện thuận lợi nhất, gồm các hoạt động như:

- Lập kế hoạch sắp xếp và theo dõi tình hình điều động tàu ra vào cảng.

- Quản lý và khai thác các phương tiện trong việc ra vào các cảng có thể sử dụng phương tiện hỗ trợ vận tải thủy, đồng thời tận dụng lợi thế của thủy triều để giảm việc phải chuyển tải hàng hóa đối với các tàu cỡ lớn.

- Quản lý và theo dõi tình hình cầu bến.

- Lập kế hoạch khai thác bốc xếp giải phóng tàu: sau khi tàu vào bến, cần huy động các trang thiết bị xếp dỡ phù hợp, tăng tốc độ giải phóng tàu. Xếp ca, máng làm hàng phù hợp.

- Lưu kho bãi, giao nhận hàng hóa tổng hợp, quản lý bãi hàng và các dịch vụ kho bãi. Ưu tiên việc xếp dỡ hàng hóa lên trực tiếp các phương tiện vận tải để giảm thiểu chi phí cho chủ hàng, nhưng với những trường hợp cần sử dụng kho bãi, phải có biện pháp quản lý khai thác kho bãi phù hợp, để tăng doanh thu cho cảng qua việc sử dụng kho bãi và xếp dỡ hàng hóa.

\section{Tình hình chung của nhóm ngành khai thác cảng hiện nay}

Trong xu thế hiện nay, xuất hiện nhiều nhà khai thác Cảng trên toàn cầu, xúc tiến thâm nhập vào thị trường xếp dỡ container nhằm tăng năng lực cạnh tranh thông qua việc thiết lập mạng lưới toàn cầu, xúc tiến liên kết dọc của các nhà khai thác Cảng dưới liên kết và thâu tóm, đầu tư cảng container ở các quốc gia khác thông qua liên doanh với các công ty chủ nhà, với các nhà khai thác khác hoặc với các hãng tàu.

Trong khi ây, hầu hết các cảng nội địa của nước ta có quy mô nhỏ, dịch vụ đơn lẻ, tính tích hợp chưa cao. Do đó, doanh nghiệp chỉ cung cấp những dịch vụ đơn giản như: làm thủ tục hải quan, cho thuê phương tiện vận tải, kho bãi, bốc xếp hàng container và một số dịch vụ khác. Bên cạnh đó, trình độ nhân lực chưa chuẩn hóa, ứng dụng công nghệ ở mức thấp, thiếu hụt trầm trọng về số lượng nhân lực có trình độ quản lý lĩnh vực logistics cũng như trình độ ngoại ngữ cũng là lí do giảm khả năng cạnh tranh của cảng nội.

\section{Thực trạng công tác kế toán quản trị tại nhóm Doanh nghiệp khai thác cảng}

- Công tác nhận diện và phân tích chi phí chưa thực sự được các doanh nghiệp quan tâm thực hiện.

- Việc xây dựng quản lý và sử dụng hệ thống định mức, dự toán thực tế chủ yếu là do các bộ phận chức năng thực hiện như: bộ phận kế hoạch, kỹ thuật, vật tư mà chưa có sự tham gia hiệu quả của bộ phận kế toán.

- Các vấn đề thuộc về tổ chức và xử lý thông tin kế toán theo góc độ của kế toán quản trị chi phí, giá thành chưa được triển khai thực hiện. 


\subsection{Mẫu nghiên cứu}

Kết quả mẫu chính thức cho thấy các đối tượng tham gia cuộc khảo sát là Lãnh đạp cấp cao (Giám đốc/Phó $\mathrm{GĐ}, \mathrm{CEO}, \mathrm{CFO}, \ldots$ ) chiểm tỷ lệ là $19.4 \%$, Kế toán trưởng/Trưởng/Phó phòng kế toán tài chính (chiếm $29.9 \%$ ), Nhân viên kế toán (kế toán tài chính, kế toán quản trị, kế toán tổng hợp) chiếm $38.9 \%$ và đối tượng khác có tham gia trả lời phiếu khảo sát chỉ chiểm một tỷ lẹ̣ nhỏ (chiếm 11.8\%).

Bảng 2. Tỷ lệ các đối tượng tham gia khảo sát

\begin{tabular}{lcc}
\hline \hline \multicolumn{1}{c}{ Đối tượng khảo sát } & Số phiếu & Tỷ lệ \% \\
\hline \hline Lãnh đạo cấp cao & 41 & $19.4 \%$ \\
KTT/Trưởng/phó phòng & 63 & $29.9 \%$ \\
Nhân viên kế toán & 82 & $38.9 \%$ \\
Khác & 25 & $11.8 \%$ \\
\hline \hline
\end{tabular}

Nguồn: Tác giả tổng hợp

Kết quả thống kê mô tả, cũng cho thấy hầu hết các nội dung (biến quan sát của nhân tố phụ thuộc) đều đạt chỉ số (mức độ đồng ý) khá thấp. Điều này cho thấy mô hình $\mathrm{ABC}$ còn khá mới lạ đối với các doanh nghiệp khai thác Cảng của Việt Nam. Chính vì vậy, việc nghiên cứu và vận dụng mô hình $\mathrm{ABC}$ chưa được triển khai hoặc vận dụng chưa hiệu quả.

Bảng 3. Tổng hợp thông tin các thang đo của nhân tố phụ thuộc

\begin{tabular}{cccccc}
\hline $\begin{array}{c}\text { Biến phụ thuộc } \\
(\mathbf{Y})\end{array}$ & $\begin{array}{c}\text { Giá trị nhỏ } \\
\text { nhất }\end{array}$ & $\begin{array}{c}\text { Giá trị trung } \\
\text { bình }\end{array}$ & Giá trị lớn nhất & Số mode & Số trung vị \\
\hline \hline $\mathrm{Y}_{1}$ & 1 & 2.56 & 5 & 1 & 3 \\
\hline $\mathrm{Y}_{2}$ & 1 & 3.09 & 5 & 2 & 3 \\
\hline $\mathrm{Y}_{3}$ & 1 & 3.46 & 5 & 4 & 4 \\
\hline $\mathrm{Y}_{4}$ & 1 & 2.79 & 5 & 3 & 3 \\
\hline $\mathrm{Y}_{5}$ & 2 & 3.08 & 5 & 2 & 3 \\
\hline $\mathrm{Y}_{6}$ & 1 & 3.63 & 5 & 3 & 3 \\
\hline \hline
\end{tabular}

Nguồn: Tác giả tổng hợp

Trong đó, $\mathrm{Y}_{1}$ - Không sử dụng thông tin $\mathrm{ABC}$ khi ra quyết định; $\mathrm{Y}_{2}$ - Có nghiên cứu nhưng không ứng dụng vào thực tế tại doanh nghiệp; $Y_{3}$ - Không quan tâm tới $A B C$ và lợi ích nó mang lại; $Y_{4}$-Sử dụng thông tin $\mathrm{ABC}$ phục vụ mục tiêu thuế, báo cáo tài chính và kiểm toán; $\mathrm{Y}_{5}$ : Không thực hiện $\mathrm{ABC}$ vì lo ngại chi phí phát sinh lớn trong quá trình thực hiện; $\mathrm{Y}_{6}$ : Từ chối sử dụng mô hình $\mathrm{ABC}$.

\subsection{Kiểm tra độ tin cậy của thang đo}

Kết quả nghiên cứu sau khi loại bỏ các biến không phù hợp từ nghiên cứu sơ bộ cho thấy mức độ tin cậy của các biển đưa vào đều đạt trên mức chuẩn. Hệ số Cronback's Alpha của tất cả các thang đo đều $>$ 0.6. Hệ số tương quan với biến tổng của tất cả các nhân tố trong thang đo đều $>0.3$. Điều này chứng tỏ thang đo được sử dụng trong mô hình nghiên cứu là phù hợp và thích hợp đưa vào phân tích những bước tiếp theo như phân tích EFA. Sau đây là kết quả tổng hợp hệ số tin cậy và phương sai trích của các thành phần (bảng 4). 

ABC (ACTIVITY BASED COSTING) CHO NHÓM CÁC DOANH NGHIÊP KHAI THÁC CẢNG CỦA VIỆT NAM

Bảng 4. tổng hợp hệ số tin cậy và phương sai trích của các thành phần

\begin{tabular}{|c|c|c|c|}
\hline & Thành phần & $\begin{array}{l}\text { Độ tin cậy thang đo } \\
\text { (Cronbach's Alpha) }\end{array}$ & $\begin{array}{l}\text { Phương sai trích } \\
\text { (\%) }\end{array}$ \\
\hline СCTC & $\begin{array}{l}\text { Cơ cấu tổ chức chưa thay đổi phù hợp để triển khai mô } \\
\text { hình } \mathrm{ABC}\end{array}$ & .934 & 83.936 \\
\hline UHCC & Thiếu sự ủng hộ của lãnh đạo & .858 & 66.642 \\
\hline HCKT & Hạn chế về kỹ thuật vận dụng & .951 & 87.111 \\
\hline NL & Thiếu nguồn lực & .803 & 63.272 \\
\hline TLNV & Tâm lý hạn chế thay đổi & .959 & 96.089 \\
\hline XDMT & $\begin{array}{l}\text { Không xây dựng mục tiêu cho từng giai đoạn triển khai } \\
\text { gắn với khen thưởng hợp lý }\end{array}$ & .955 & 95.654 \\
\hline NT & Hạn chế về nhận thức & .810 & 57.019 \\
\hline Y & Hạn chế vận dụng mô hình $\mathrm{ABC}$ vào doanh nghiệp & .871 & 72.486 \\
\hline
\end{tabular}

Nguồn: Tác giả tổng hợp tù phân tích trên SPSS

\subsection{Phân tích EFA}

Kết quả kiểm định Barlett cho thấy giữa các biến trong tổng thể có mối tương quan với nhau (sig = $0.000<0.05)$. Đồng thời, hệ số $\mathrm{KMO}=.779(>0.5)$, chứng tỏ phân tích nhân tố để nhóm các biến lại với nhau là thích hợp và dữ liệu phù hợp cho việc phân tích nhân tố (bảng 5).

Bảng 5. Kết quả kiểm tra KMO và kiểm định Barlett

\begin{tabular}{lll}
\hline \hline Hệ số KMO (Kaiser-Meyer-Olkin) & & .779 \\
\hline Mô hình kiểm tra của Bartlett & Giá trị Chi-Square & 4911.100 \\
\cline { 2 - 3 } & Bậc tự do & 325 \\
\cline { 2 - 3 } & Sig (giá trị P - value) & .000 \\
\hline \hline
\end{tabular}

Nguồn: Tác giả phân tích trên SPSS

Kết quả phương sai trích đạt $77.75 \%$ ( $>50 \%)$. Hệ số tải nhân tố (factor loading) của các biến đều đạt yêu cầu $(>0.5)$. Đây cũng là lần phân tích nhân tố cuối cùng và 26 biến này được xem xét kết quả rút trích nhân tố ở các bước tiếp theo. Kết quả bảng 6 cho thấy, theo tiêu chuẩn Eigenvalue $>1$ thì có 7 nhân tố được rút ra và 7 nhân tố này sẽ giải thích được $77.75 \%$ biến thiên của dữ liệu. Kết quả phân tích ta được ma trận xoay nhân tố như bảng 6 dưới đây. 
Bảng 6. Kết quả phân tích nhân tố EFA

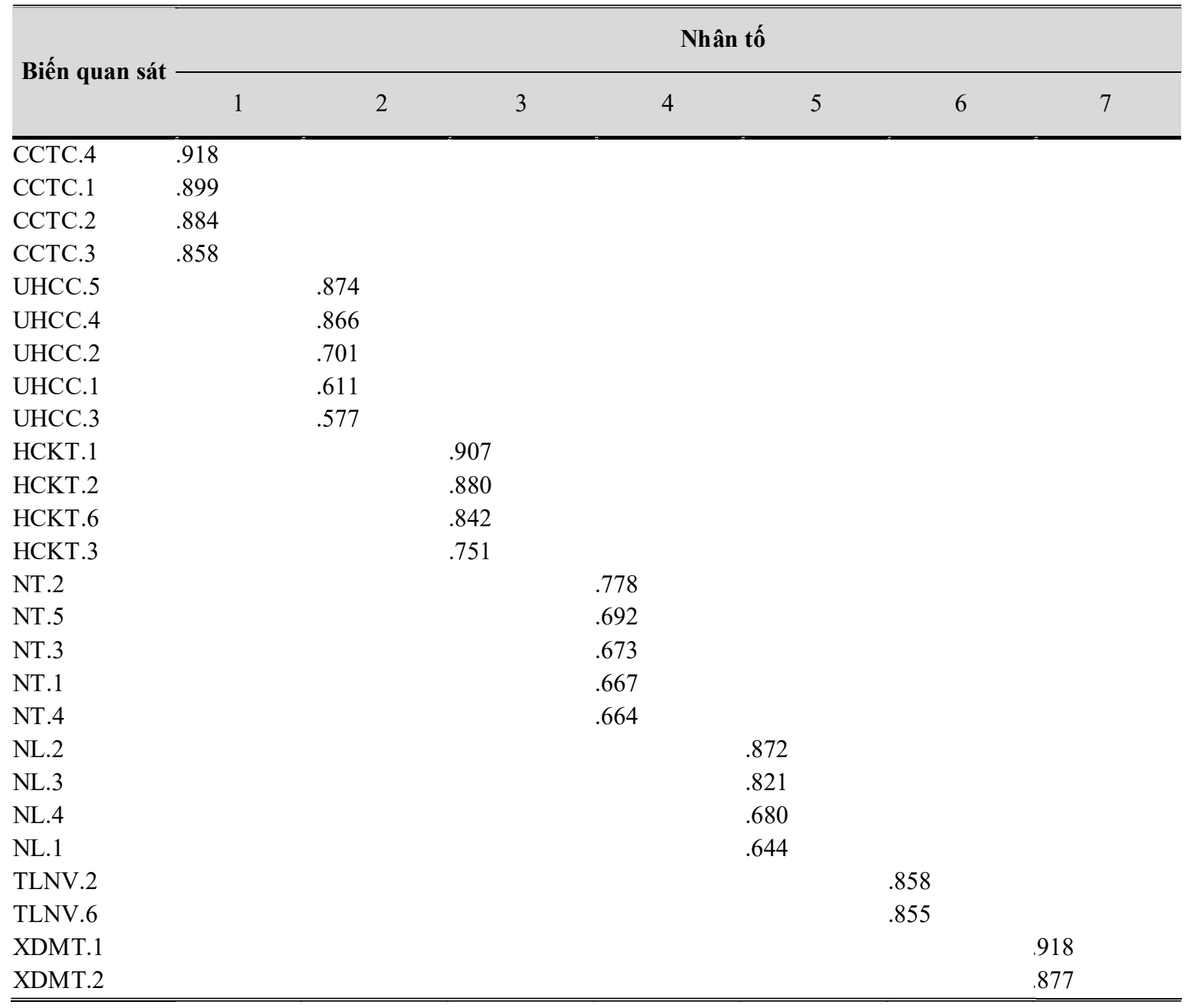

Nguồn: Tác giả phân tích trên SPSS

\subsection{Phân tích hồi quy đa biến}

Kết quả kiểm định ANOVA với mức ý nghĩa $1 \%(>\operatorname{sig}=0.000)$ cho thấy mô hình hồi quy tuyến tính bội đã xây dựng phù hợp với dữ liệu đã thu thập (bảng 7).

Bảng 7. Kết quả phân tích ANOVA

\begin{tabular}{clccccc}
\hline \multirow{2}{*}{ Mô hình } & $\begin{array}{c}\text { Tổng bình } \\
\text { phương }\end{array}$ & Bậc tự do & $\begin{array}{c}\text { Trung bình bình } \\
\text { phương }\end{array}$ & F & Sig. \\
\hline \hline \multirow{2}{*}{1} & Hồi quy & 180.880 & 7 & 25.840 & 180.135 & .000 \\
& Phần dư & 29.120 & 203 & .143 & & \\
& Tổng & 210.000 & 210 & & \\
\hline \hline
\end{tabular}

Nguồn: Tác giả tổng hợp phân tích trên SPSS

Mô hình nghiên cứu ban đầu đã được Tác giả điều chỉnh sau khi có kết quả phân tích nhân tố khám phá, mô hình chính thức đưa vào phân tích hồi quy tuyến tính bội được thể hiện như sau (biến CLKD bị loại bỏ):

$$
\mathrm{Y}=\beta 0+\beta 1 * \mathrm{CCTC}+\beta 2 * \mathrm{UHCC}+\beta 3 * \mathrm{HCKT}+\beta 4 * \mathrm{NT}+\beta 5 * \mathrm{NL}+\beta 6 * \mathrm{TLNV}+\beta 7 * \mathrm{XDMT}+\varepsilon
$$


Trong đó:

- Biến phụ thuộc $\mathrm{Y}$ : hạn chế vận dụng mô hình $\mathrm{ABC}$ vào nhóm doanh nghiệp khai thác Cảng tại Việt Nam.

- Biến độc lập: gồm 7 biến là (1) Cơ cấu tổ chức, (2) ủng hộ cấp cao, (3) Hạn chế kỹ thuật, (4) Nhận thức, (5) Nguồn lực, (6) Tâm lý nhân viên, (7) Xây dựng mục tiêu.

Bảng 8 . Kết quả phân tích hồi quy đa biến

\begin{tabular}{|c|c|c|c|c|c|c|c|}
\hline \multirow{2}{*}{ Mô hình } & \multicolumn{2}{|c|}{ Hệ số chưa chuẩn hóa } & \multirow[t]{2}{*}{ Hệ số chuẩn hóa } & \multirow{2}{*}{$\mathbf{t}$} & \multirow{2}{*}{ Sig. } & \multicolumn{2}{|c|}{$\begin{array}{c}\text { Thống kê đa cộng } \\
\text { tuyến }\end{array}$} \\
\hline & B & $\begin{array}{l}\text { Sai số } \\
\text { chuẩn }\end{array}$ & & & & $\begin{array}{c}\text { Hệ số } \\
\text { Tolerance }\end{array}$ & VIF \\
\hline (Constant) & .000 & .026 & & .000 & 1.000 & & \\
\hline ССТC & .154 & .026 & .154 & 5.902 & .000 & 1.000 & 1.000 \\
\hline UHCC & .630 & .026 & .630 & 24.095 & .000 & 1.000 & 1.000 \\
\hline HCKT & .578 & .026 & .578 & 22.111 & .000 & 1.000 & 1.000 \\
\hline NT & .189 & .026 & .189 & 7.250 & .000 & 1.000 & 1.000 \\
\hline NL & .145 & .026 & .145 & 5.542 & .000 & 1.000 & 1.000 \\
\hline TLNV & .165 & .026 & .165 & 6.312 & .000 & 1.000 & 1.000 \\
\hline XDMT & .151 & .026 & .151 & 5.792 & .000 & 1.000 & 1.000 \\
\hline
\end{tabular}

Nguồn: Tác giả tổng hợp phân tích trên SPSS

Trong bảng 8 , khi so sánh mức ý nghĩa $\alpha=1 \%$ với giá trị Sig. của các biến (7 thành phần) gồm: (1) Cơ cấu tổ chức - CCTC, (2) ủng hộ cấp cao - UHCC, (3) Hạn chế kỹ thuật - HCKT, (4) Nhận thức - NT, (5) Nguồn lực - NL, (6) Tâm lý nhân viên - TLNV, (7) Xây dựng mục tiêu - XDMT, kết quả cho thấy hệ số của các biến đều có ý nghĩa thống kê (giá trị Sig. $<1 \%$ ) và đạt được độ tin cậy cao. Ngoài ra, hệ số VIF đều nhỏ (nhỏ hơn 10) và hệ số Tolerance đều $>0,5$ cho thấy không có hiện tượng đa cộng tuyến xảy ra [17].

Kết quả mô hình hồi quy bội được thể hiện như sau:

$\mathrm{Y}=0.154 \mathrm{CCTC}+0.630 \mathrm{UHCC}+0.578 \mathrm{HCKT}+0.189 \mathrm{NT}+0.145 \mathrm{NL}+0.165 \mathrm{TLNV}+0.151 \mathrm{XDMT}$

Đánh giá mức độ giải thích của mô hình

Bảng 9. Đánh giá mức độ phù hợp của mô hình hồi quy tuyến tính đa biến

\begin{tabular}{|c|c|c|c|c|c|c|c|c|}
\hline \multirow{2}{*}{$\begin{array}{r}\text { Mô } \\
\text { hình }\end{array}$} & \multirow{2}{*}{$\begin{array}{c}\text { Hệ số } \\
\text { R }\end{array}$} & \multirow{2}{*}{$\begin{array}{c}\text { Hệ số } \\
\text { R }^{2} \text { hiệu } \\
\text { chỉnh }\end{array}$} & \multirow{2}{*}{$\begin{array}{c}\text { Sai số chuẩn } \\
\text { của ước } \\
\text { lượng }\end{array}$} & \multicolumn{4}{|c|}{ Thống kê thay đổi } & \multirow{2}{*}{$\begin{array}{c}\text { Hệ số } \\
\text { Durbin- } \\
\text { Watson }\end{array}$} \\
\hline & & & & Hệ số R² & Hệ số F & $\begin{array}{c}\text { Bậc tự } \\
\text { do } 1\end{array}$ & $\begin{array}{c}\text { Bậc tự } \\
\text { do } 2\end{array}$ & \\
\hline 1 & $.928^{\mathrm{a}}$ & .857 & .3787 & .861 & 180.135 & 7 & $203^{a}$ & 2.45 \\
\hline
\end{tabular}


a. Biến độc lập: (Hằng số) CCTC, UHCC, HCKT, NT, NL, TLNV, XDMT

b. Biến phụ thuộc : Y

Từ bảng 9 cho thấy, giá trị hệ số tương quan là $0.928>0.5$. Do vậy, đây là mô hình thích hợp để sử dụng đánh giá mối quan hệ giữa biến phụ thuộc và các biến độc lập. Hệ số xác định của mô hình hồi quy $\mathrm{R}^{2}$ điều chỉnh là 0.857 . Điều này cho biết khoảng $85,7 \%$ sự biến thiên các nhân tố ảnh hưởng đến việc vận dụng mô hình kế toán quản trị $\mathrm{ABC}$ vào nhóm các doanh nghiệp khai thác Cảng tại Việt Nam có thể giải thích được từ mối quan hệ tuyến tính giữa biến $Y$ với các biến độc lập.

Hệ số tương quan của biến phụ thuộc với từng biến độc lập dao động từ 0.154 đến 0.63 (mức tương quan trung bình đến tương quan trung bình khá). Trên thực tế, với mức ý nghĩa $1 \%$, giả thuyết hệ số tương quan của tổng thể bằng 0 bị bác bỏ. Điều này có nghĩa là trong tổng thể, tồn tại mối tương quan tuyến tính (ảnh hưởng) giữa biến phụ thuộc - hạn chế vận dụng phương pháp kế toán quản trị $\mathrm{ABC}$ vào nhóm doanh nghiệp khai thác Cảng tại Việt Nam (Y) với các biến độc lập.

+ Kiểm tra phương sai thay đổi Đồ thị phần dư chuẩn hoá phân tán ngẫu nhiên quanh trục 0 , do đó ta có thể kết luận rằng không có hiện phương sai thay đổi [17].

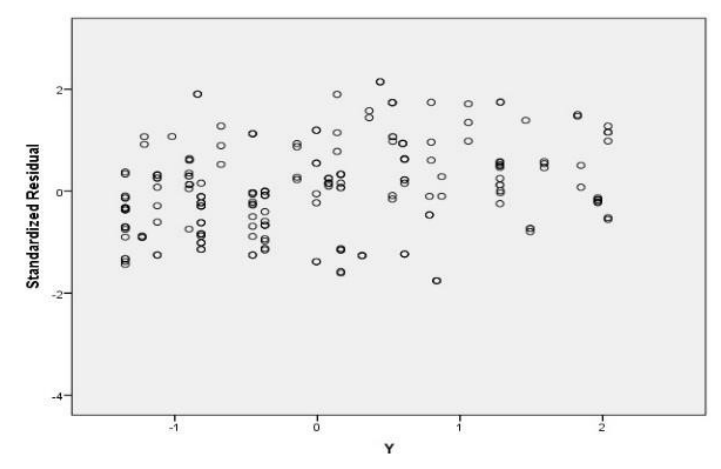

Hình 10: Đồ thị phân tán phần dư

+ Kiểm tra hiện tượng tư tuoong quan: Kết quả phân tích trên (bảng 9) cho thấy hệ số Durbin-Watson $\mathrm{d}=2.45(1<\mathrm{d}<3)$, nên mô hình không xảy ra hiện tượng tư tương quan [17].

\subsection{Thảo luận}

Sau khi phân tích dữ liệu và đưa ra kết quả nghiên cứu về mức độ ảnh hưởng của các nhân tố tới việc triển khai mô hình kế toán quản trị $\mathrm{ABC}$ vào nhóm doanh nghiệp khai thác cảng tại Việt Nam, có thể thấy rằng muốn triển khai mô hình được hiệu quả không bị gián đoạn thì điều quan trọng nhất chính là phía nhà lãnh đạo công ty, là người đứng đầu và có quyền quyết định bất kỳ vấn đề tại doanh nghiệp. Kế tiếp là nâng cao và hỗ trợ kỹ thuật vận dụng trong quá trình thu thập và phân tích dữ liệu khảo sát. Nếu doanh nghiệp đáp ứng hai yếu tố trên, khả năng thành công của mô hình tương đối cao. So sánh với mô hình nghiên cứu của các tác giả Nguyễn Việt Hưng \& Trần Thứ $\mathrm{Ba}(2016)$, Nhân tố ảnh hưởng đến việc hạn chế vận dụng phương pháp ABC trong kế toán quản trị tại các doanh nghiệp Việt Nam thì mức độ tác động và số lượng nhân tố ảnh hưởng tới mô hình xét tổng thể có phần tương đồng khá lớn. Tuy nhiên, hai đối tượng nghiên cứu của hai đề tài khác nhau, một là nhóm doanh nghiệp khai thác cảng (tác giả), còn một bên thì nghiên cứu chung cho các doanh nghiệp Việt Nam. Vì vậy, tỷ lệ tác động của các nhân tố và số lượng nhân tố trong mô hình cũng có phân hơi khác nhau bảng 11). 
ABC (ACTIVITY BASED COSTING) CHO NHÓM CÁC DOANH NGHIÊP KHAI THÁC CẢNG CỦA VIỆT NAM

Bảng 11. So sánh hệ số ảnh hưởng với nghiên cứu khác

\begin{tabular}{|c|c|c|}
\hline Nhân tố & $\begin{array}{c}\text { Nguyễn Việt Hưng \& Trần } \\
\text { Thứ Ba (2016) }\end{array}$ & Tác giả \\
\hline Thiếu sự ủng hộ của lãnh đạo & 0.103 & 0.63 \\
\hline Hạn chế về kỹ thuật triển khai & 0.639 & 0.578 \\
\hline Tâm lý e ngại của nhân viên & 0.577 & 0.165 \\
\hline Chiến lược kinh doanh & 0.234 & Không có \\
\hline Hạn chế về nguồn lực & 0.136 & 0.145 \\
\hline Cấu trúc tổ chức không phù hợp & 0.122 & 0.154 \\
\hline Chưa xây dựng mục tiêu & 0.134 & 0.151 \\
\hline Hạn chế về nhận thức & 0.150 & 0.189 \\
\hline Chưa quan tâm huấn luyện & 0.137 & Không có \\
\hline
\end{tabular}

Nguồn: tác giả tổng hợp

\section{KẾT LUẬN VÀ KIẾN NGH!}

Qua kết quả nghiên cứu đã loại một biến là" Chiến lược kinh doanh". Vì cơ bản, trong biến "Xây dựng mục tiêu" đã bao hàm cả chiến lược kinh doanh. Vì vậy, việc vận dụng mô hình $\mathrm{ABC}$ vào nhóm doanh nghiệp khai thác Cảng tại Việt Nam chịu ảnh hưởng bởi 7 biến độc lập gồm: ủng hộ của lãnh đạo cấp cao, kỹ thuật triển khai, nguồn lực, nhận thức, cơ cấu tổ chức, xây dựng mục tiêu, tâm lý nhân viên. Đặc biệt là nhân tố ủng hộ của cấp cao và kỹ thuật triển khai mô hình với hệ số khá cao.

Dựa vào kết quả nghiên cứu, để một doanh nghiệp khai thác Cảng có thể vận dụng tốt và hiệu quả mô hình kế toán quản trị theo phương pháp $\mathrm{ABC}$ thì trước tiên, nhà quản trị phải thay đổi nhận thức và có thái độ khuyến khích động viên cho nhân viên, tạo điều kiện thuận lợi để thực hiện mô hình tốt nhất. Đồng thời, có cái nhìn toàn diện và tổng thể, đưa ra những lựa chọn phù hợp với năng lực và quy mô của doanh nghiệp. Nên cân nhắc trước những quyết định giữa cơ hội của sự đánh đổi giữa các dự án/hoạt động, cắt giảm các chi phí không cần thiết và các dự án/hoạt động không đem lại hiệu quả cho đơn vị mà thay vào đó là xây dựng mô hình kế toán quản trị $\mathrm{ABC}$ phù hợp với cơ cấu đem lại kết quả tối ưu nhất.

Vấn đề hạn chế về kỹ thuật triển khai $\mathrm{ABC}$ dường như là vướng mắc lớn đối với các doanh nghiệp ban đầu triển khai $\mathrm{ABC}$. Chính vì vậy, câu hỏi lớn đặt ra khi triển khai mô hình $\mathrm{ABC}$ tại các doanh nghiệp khai thác cảng là làm thế nào để xây dựng mô hình một cách hiệu quả. Câu trả lời rất đơn giản là khi nhận diện số lượng hoạt động hợp lý, gộp các hoạt động, lựa chọn tiêu thức phù hợp thì mô hình sẽ cho đạt hiệu quả. Thế nhưng, đó là lý thuyết là câu trả lời cửa miệng mà bất kỳ ai cũng có thể nói được. Vấn đề lớn ở đây là làm sao để từ cái lý thuyết đem áp dụng vào thực tế đạt mức độ tương đối hợp lý là điều khó khăn đối với các doanh nghiệp hiện nay không chỉ Việt Nam mà các nước trên thế giới cũng đang gặp phải.

Vì vậy, trước hết doanh nghiệp cần đào tạo các đội ngũ nhân viên hiểu bản chất thật sự của vấn đề của phương pháp $\mathrm{ABC}$. Xây dựng quy trình hoạt động của công ty một cách chi tiết và hợp lý nhất để các nhân viên thực hiện hiểu rõ tính chất của các hoạt động từ đó lựa chọn các hoạt động chính trong quá trình tiến hành vận dụng mô hình. Mặc khác, cần có công tác phỏng vấn nhân viên liên quan trực tiếp tới các hoạt động chính để xác định hợp lý nguồn gốc phát sinh chi phí và lựa chọn tiêu thức phân bổ phù hợp với từng hoạt động, chẳng hạn đối với hoạt động dỡ hàng từ cảu bờ xuống xe container thì dựa vào số lần cẩu hay số lượng cont để lựa chọn tiêu thức một cách hợp lý. Một vấn đề cần lưu ý, khi thực hiện phỏng vấn nhân viên để đảm bảo độ tin cậy của thông tin nhóm thực hiện cần phỏng vấn những nhân viên làm việc lâu năm và nên trực tiếp quan sát lại, để tin chắc rằng thông tin mình nhận được là chính xác 
Tuy nhiên, việc thu thập và xử lý thông tin vô cùng quan trọng và ảnh hưởng tới kết quả của việc ứng dụng mô hình có hiệu quả hay không. Để đảm bảo điều đó, có ba phương pháp với mức độ chính xác về dữ liệu có thể được sử dụng là: ước đoán, đánh giá hệ thống, thu thập dữ liệu thực tế.

Trường hợp không thể có số liệu thực tế hoặc tốn quá nhiều chi phí thì có thể ước đoán để tính ra các tỷ lệ cho giai đoạn 1 và giai đoạn 2 , tức là tỷ lệ phân bố các chi phí vào các hoạt động và từ các hoạt động vào sản phẩm. Tuy nhiên, phương pháp này đòi hỏi cần sự kết hợp của nhiều nhóm và bộ phận với nhau và kiến thức của họ về trung tâm chi phí, để dưa ra một con số ước đoán hợp lý nhất. Đối với phương pháp đánh giá hệ thống, việc tính toán các tỷ lệ này dựa trên kỹ thuật hệ thống, chẳng hạn sử dụng phương pháp phân tích thứ bậc AHP (Anlynic Hierachical Process). Thông qua việc đặt câu hỏi và phỏng vấn, $\mathrm{AHP}$ sẽ dựa vào kết quả phỏng vấn để phân tích ra phần trăm chi phí và phân bổ cho từng hoạt động và sản phẩm. Và cuối cùng, phương pháp thu thập trực tiếp cho kết quả chính xác nhất tuy nhiên tốn nhiều chi phí. Vì vậy, để thu thập thông tin mang tính tương đối, cần cân nhắc nên áp dụng phương pháp nào hợp lý. Đối với phương pháp ước đoán nên sử dụng đối với các chi phí dưới $10 \%$ và mức độ tác động không lớn tới tổng thể.

Thêm vào đó, nhân viên là người thực hiện trong quá trình triển khai dự án, chính vì thế tâm lý của nhân viên ảnh hưởng đáng kể tới quá trình triển khai mô hình có thành công hay không. Vì vậy, trước khi thực hiện và triển khai mô hình, cấp lãnh đạo cần động viên tinh thần nhân viên và đảm bảo rằng các nhân viên không bị ảnh hưởng khi có những sai sót lúc triển khai, tuy nhiên nếu như vậy sẽ làm nhân viên mang tâm lý ỷ lại không tập trung làm việc vì không phải chịu trách nhiệm đối với cồng việc của mình. Do đó, cần có những phần thưởng mang tính vật chất đối với các nhân viên thực hiện tốt trong quá trình làm việc, như vậy bản thân nhân viên sẽ tự giác và có động lực hơn trong làm việc. Đó cũng là hình thức duy trì mối quan hệ xã hội trong doanh nghiệp mà nhiều tập đoàn lớn áp dụng để tạo sự hòa hợp giữa nhân viên với công ty.

Ngoài ra, đơn vị nên tổ chức các buổi thảo luận với nhân viên để hiểu được những gì mà họ muốn nhằm kịp thời hỗ trợ và nâng cao chất lượng đội ngũ nhân viên trong công ty hơn. Trong quá trình triển khai, vẫn có một số nhân viên không tuân thủ những quy định làm việc không hiệu quả, có tâm lý ỷ lại thì nên xem xét để thay thế bằng đội ngũ nhân viên có trách nhiệm và năng lực tốt hơn.

Một thực tế hiện nay, nhiều doanh nghiệp khi thực hiện xong mô hình ở giai đoạn đầu cảm thấy hiệu quả, tuy nhiên tới các giai đoạn sau thì mức độ kỳ vọng giảm đi so với mong đợi ban đầu kế hoạch, chính vì vậy mà vội "cất" mô hình đi. Lí do của vấn đề này là doanh nghiệp chưa hiểu rõ cơ chế hoạt động của mô hình, bất kỳ một vấn đề nào cũng vậy, khi yếu tố bên ngoài thay đổi, chắc chắn sẽ tác động tới mô hình hiện tại. Vì vậy, trong từng giai đoạn đòi hỏi doanh nghiệp phải thay đổi và xây dựng mục tiêu cho hợp lý. Vấn đề thay đổi và xây dựng mục tiêu cần phải chọn thời điểm, khi có những biến động hay thay đồi lớn trong cơ cấu tổ chức hay tỷ trọng chi phí trong doanh nghiệp thì phải xét lại các hoạt động chính và lực chọn các tiêu thức phân bổ hợp lý hơn. Không nên có suy nghĩ khuôn mẫu trong bất kỳ một mô hình nào, công việc này nên định kỳ để mô hình $\mathrm{ABC}$ triển khai phù hợp cho từng giai đoạn của hoạt động trong doanh nghiệp. Dưới đây là quy trình thực hiện cơ bản cho nhóm các doanh nghiệp khai thác Cảng tại Việt Nam:

Quy trình thực hiện gồm 3 giai đoạn:

- Giai đoạn chuẩn bị

- Giai đoạn thực hiện

- Giai đoạn nâng cao cải thiện phương pháp

\section{- Giai đoạn chuẩn bi}

Trong giai đoạn này doanh nghiệp cần phải xác định được ngồn lực hiện có của doanh nghiệp (Con người, công nghệ, các phương án hoạt động, ...) từ đó tổ chức và phân bổ nguồn lực cần thiết để đạt mục tiêu phù hợp với doanh nghiệp. Đồng thời, cần lập một đội thực hiện $\mathrm{ABC}$ để thực hiện nhiệm vụ của mình theo nhiều cách, phụ thuộc vào sự sẳn có của nguồn lực, thời gian và yêu cầu bảo đảm hoạt động hàng ngày. Để đảm bảo thực hiện $\mathrm{ABC}$ hiệu quả, cơ cấu thích hợp nhất là đội gồm những người năm giữ các vị trí chủ chốt tại các bộ phận, có trình độ cao trong lĩnh vực chuyên môn và cam kết đến kết quả cuối cùng của công việc. Trong trường hợp thiếu kinh nghiệm về $\mathrm{ABC}$, đội cần nhờ tới các chuyên gia tư vấn trong quá trình thực hiện. 


\section{- Giai đoạn thưc hiện}

Trong giai đoạn này, cần xem xét các đặc điểm cơ bản của doanh nghiệp và thu thập tài liệu chi tiết về cách thức doanh nghiệp vận hành để hiểu rõ và xây dựng chi tiết quá trình hoạt động của doanh nghiệp. Sau đây là 4 bước cơ bản quá trình vận dụng mô hình $\mathrm{ABC}$ vào doanh nghiệp:

- Phân tích quá trình sản xuất thành các hoạt động

- Xác định các trung tâm hoạt động

- Xác định chi phí cho các trung tâm hoạt động và các hoạt động

- Phân bổ chi phí cho từng đối tượng tính phí.

$\mathrm{B} 1$ : Qúa trình thực hiện mô hình $\mathrm{ABC}$ bắt đầu bằng việc phân tích quá trình sản xuất của doanh nghiệp. Qua đó, xác định được các hoạt động và quá trình chủ yếu trong tổ chức như thời gian, chất lượng, tiến độ thực hiện, các đầu ra và đầu vào cần thiết cho mỗi hoạt động. Qua đó nhận diện được các hoạt động nào làm tăng giá trị, hoạt động nào không tạo thêm giá trị. Bước này rất quan trong, nó là bước cơ sở để xác định trung tâm chi phí cho bước tiếp theo.

B2: Sau khi xác định được các hoạt động tiêu dùng nguồn lực, KTQT phải xác định các trung tâm hoạt động. Mặc dù về cơ bản, không nhất thiết phải xác định trung tâm hoạt động, tuy nhiên số lượng hoạt động tại các doanh nghiêp khai thác Cảng khá nhiều, vì vậy để đơn giản cho công việc, cần nhóm lại các hoạt động có cùng bản chất xét ở khía cạnh nguyên nhân phát sinh chi phí.

B3: Xác định chi phí cho các trung tâm hoạt động và các hoạt động:

Dựa vào sổ sách kế toán để xác định tổng chi phí của các nguồn lực như: Khấu hao tài sản, Chi phí vận chuyển, nhiên liệu, ... tiếp đó chi phí sẽ được quy nạp cho các trung tâm hoạt động đã được xác định ở trên bước 2 , sau đó được phân bổ cho các trung tâm dựa theo các tiêu thức phân bổ thích hợp.

B4: Phân bổ chi phí cho từng đối tượng tính phí (dịch vụ, khách hàng, ...)

Để lựa chọn một khía canh hay một nhân tố nào đó của hoạt động làm nguồn phát sinh chi phí cho từng hoạt động để làm cơ sở để phân bồ chi phí cho từng dịch vụ cần cân nhắc hai nhân tố sau đây:

Kế toán quản trị phải có dữ liệu phong phú về nguồn phát sinh chi phí định lựa chọn.

Nguồn phát sinh chi phí phải phản ánh được mức độ tiêu dùng các hoạt động của từng loại dịch vụ

Sau khi đã xác định được nguồn phát sinh kết hợp với dữ liệu về mức độ tiêu dùng các hoạt động ta xác định tỉ lệ phân bổ tương ứng cho từng sản phẩm thông qua 3 phương pháp cơ bản như đã nói là:

Ước đoán, đánh giá hệ thống bằng việc dựa vào kỹ thuật $\mathrm{AHP}$, khảo sát thực tế.

Đồng thời, trong bượ 3 và bước 4 ta có thể thiếp lập ma trận Chi Phí-Hoạt động $(E A D)$ và Họa động-Dịch vụ (APD) để xác định chi phí cho từng hoạt động (dịch vụ).

\section{- Giai đoạn nâng cao cải tiến phương pháp}

Các hoạt động của doanh nghiệp luôn thay đổi theo từng hoàn cảnh khác nhau, với nhiêu yếu tố tác động liên tục, do đó không thể áp dụng một mô hình cố định trong thời gian dài. Mô hình $\mathrm{ABC}$ đòi hỏi phải được thiết kế linh hoạt để có thể điều chỉnh thuận tiện theo từng yếu tố môi trường tác động. Vì vậy, sau khi hoạt động các cấp lãnh đạo phải thường xuyên thẩm định lại chiến lược, cập nhật nhân tố và các vấn đề kinh doanh chủ yếu liên quan tới đối tượng chịu phí, cụ thể:

Xem xét giữa số liệu thực tế và kế hoạch để tìm ra các vấn đề còn bất hợp lý.

Xem xét lại phương pháp $\mathrm{ABC}$ có thể phối hợp phương pháp $\mathrm{T}$. $\mathrm{ABC}$ và $\mathrm{VCP}$ nhằm đạt hiệu quả cao trong hoạt động.

\section{TÀI LIỆU THAM KHẢO}

[1] Kaplan S. Robert \& Robin Cooper (1998), Cost \& Effect, Using Integrated Cost System to Drive Profitability and Performance, Boston: Harvard Business School Press.

[2] Cooper Robin \& Robert S. Kaplan (1991), Profit priorities from Activity - Based Costing, Harvard Business Review.

[3] Owen P. Hall, Charles J. Mc. Peak (2011), Are SMEs ready for ABC, Journal of Accounting and Finance, Vol.11(4), Page 11-13. 
32 GIẢI PHÁP VÀ KỸ THUẬT TRIỂN KHAI ỨNG DUUNG MÔ HİNH KÊ TOÁN QUẢN TRI

ABC (ACTIVITY BASED COSTING) CHO NHÓM CÁC DOANH NGHIÊP KHAI THÁC CẢNG CỦA VIẸTT NAM

[4] Wiriya (2002), Exploration of Factors influencing on choice the Activity-Based-Costing System in Iranian Organizations, Vol.2

[5] Manivannan (2010), The Success and Failure of Activity-Based-Costing Sytems

[6] Brent Bahnub (2012), Activity-Based-Management for Financial Institutions

[7] Douglas T. Hicks (1992), Activity-Based-Costing for small and Mid-Sized Businesses An Implementation Guied

[8] Turney (1996), Activity-Based-Cost and Environmental Management, Georgia Institut of Technology, U.S.A

[9] Johnson (1990), Pitfalls in Using ABC Cost- Driver Information to Manage operating costs

[10] Robin A.Sanford (2009), The Impact of Activity- based -Costing on Organizational Performance

[11] Nguyễn Việt Hưng\&Trần Thứ Ba (2016), Nhân tố ảnh hưởng đến sự hạn chế vận dụng mô hình $\mathrm{ABC}$ (Activity Based Costing) trong kế toán quản trị tại các doanh nghiệp Việt Nam, Tạp chí Phát triển \& Hội nhập, Số 31 (41), trang 65-70.

[12] Nguyễn Thị Ngọc Lan (2012), Tổ chưc kế toán quản trị chi phí vận tải hàng hóa trong các công ty vận tải đường bộ Việt Nam, luận án tiến sĩ, Mã số: 62.34.30.01

[13] Phạm Hồng Hải (2013), Nghiên cưu quản trị chi phí kinh doanh theo quá trình hoạt động (ABC/M) trong các doanh nghiệp chế biến gỗ Việt Nam, Luận án tiến sĩ, Mã số: 62.34.05.01.

[14] Huỳnh Tấn Dũng\&Gong Guang Ming (2013), So sánh phuơng pháp ABC và phuơng pháp tính giá truyền thống sụ cần thiết áp dụng phưong pháp ABC cho các doanh nghiệp Việt Nam, Tạp chí Đại Học Công Nghiệp, số $1(10)$.

[15] Huỳnh Tấn Dũng (2017), Giáo trình kế toán quản trị phần 1, NXB Đại học Công Nghiệp TP.HCM.

[16] Nguyễn Huy Tâm (2013), Áp dụng phương pháp ABC trong quản lý chi phí tại công ty TNHH Sản xuất BETA, Luận án mã đề tài KT.12.05

[17] Hoàng Trọng\& Chu Nguyễn Mộng Ngọc (2008), Phân tích dũ liệu nghiên cưu SPSS, NXB Hồng Đức.

[18] Hoàng Ngọc Nhậm (2008), Giáo trình Kinh tế luợng, NXB Lao động - Xã hội.

[19] Bùi Minh Nguyệt (2013), Vận dụng phuoong pháp tính giá trên cơ sở hoạt động (ABC) phục vụ cho việc ra quyết định chiến lược tại cty TNHH Ý CHÍ VIẸT, Luận án Thạch Sĩ, Mã đề tài: 60340301

Ngày nhận bài: 25/07/2017

Ngày chấp nhận đăng: 09/11/2017

C 2017 Trường Đại học Công nghiệp thành phố Hồ Chí Minh 